\title{
MULTI-UAV FLIGHT USING VIRTUAL STRUCTURE COMBINED WITH BEHAVIORAL APPROACH
}

\author{
Cezary KOWNACKI* \\ ${ }^{\star}$ Faculty of Mechanical Engineering, Bialystok University of Technology, Wiejska 45C, 15-351 Bialystok, Poland \\ c.kownacki@pb.edu.pl
}

received 8 June 2015, revised 15 March 2016, accepted 21 March 2016

\begin{abstract}
Implementations of multi-UAV systems can be divided mainly into two different approaches, centralised system that synchronises positions of each vehicle by a ground station and an autonomous system based on decentralised control, which offers more flexibility and independence. Decentralisation of multi-UAV control entails the need for information sharing between all vehicles, what in some cases could be problematic due to a significant amount of data to be sent over the wireless network. To improve the reliability and the throughput of information sharing inside the formation of UAVs, this paper proposes an approach that combines virtual structure with a leader and two flocking behaviours. Each UAV has assigned different virtual migration point referenced to the leader's position which is simultaneously the origin of a formation reference frame. All migration points create together a virtual rigid structure. Each vehicle uses local behaviours of cohesion and repulsion respectively, to track its own assigned point in the structure and to avoid a collision with the previous UAV in the structure. To calculate parameters of local behaviours, each UAV should know position and attitude of the leader to define the formation reference frame and also the actual position of the previous UAV in the structure. Hence, information sharing can be based on a chain of local peer-to-peer communication between two consecutive vehicles in the structure. In such solution, the information about the leader could be sequentially transmitted from one UAV to another. Numerical simulations were prepared and carried out to verify the effectiveness of the presented approach. Trajectories recorded during those simulations show collective, coherence and collision-free flights of the formation created with five UAVs.
\end{abstract}

Key words: Multi-UAV, Virtual Structure, Formation, Flocking Behaviors

\section{INTRODUCTION}

In the last few years, technology development in the area of unmanned aerial vehicles (UAV) is reflected in a high number of commercial flying robots, which became widely available for even inexperienced users. Therefore, taking photos or making movies from the air becomes commonplace and just popular. The main reason is a simplicity of controlling of such kind of vehicles, whose autonomy is based mainly on the use of GPS (Global Positioning System) assisted by a simple AHRS (Attitude and Heading Reference System). Hence, the autonomous flight of single UAV through the sequence of waypoints is just a trivial task in present days. But frequently, a flight of single UAV is insufficient especially, if a mission requires collecting lots of information in a short time. If we consider a relatively short flight time of electrical vehicles, multi-UAV missions seem to be the best way to achieve this. However, coordinated and collective flights are no longer so easy to prepare by the use of commonly available UAVs. Autonomous control of multi-UAV systems is much more complex and it requires applying of additional mechanisms of control and sharing of navigational parameters, which are crucial for position coordination in 3D. Those issues are not supported yet by any commercial UAV system, as that multi-UAVs systems are still under development and they are not ready to be used even for a military purpose. Therefore, there is a wide area to conduct research in this field to achieve coordinated flight of a group of UAV.

Most of known algorithms designed for the formation control could be classified to three separate subsets of approaches to multi-UAV systems, namely, bio-inspired behavioural methods (Kownacki and Ołdziej, 2015; Virágh Cs. et al., 2014, Quintero et al., 2013), virtual structure methods (Norman et al., 2008; Ren and Beard, 2004; Cai et al., 2012; Shao et al., 2014; Low et al, 2011; Askari et al., 2015; Shan et al., 2005) and leader-follower methods (Ambroziak and Gosiewski, 2014; Yun et al. 2008; Xingping et al., 2003). The first approach treats a group of UAVs like a flock of birds that behaves according to four major behaviours formulated by C. Reynolds, i.e. cohesion, repulsion, migration and alignment (Reynolds, 1987). The advantage of that approach is a decentralised control, which makes possible to self-organize a structure of the swarm through local interactions between vehicles. But the structure of the flock is not rigid, but even it has random nature. The weakness arises from the necessity of information sharing between all or at least most of flock members, which is essential to estimate parameters of flocking behaviours, especially if cohesion and migration are acting globally. The second approach allows creating a formation in the form of a virtual rigid structure, where relative locations of each vehicle should be continuously constant as it is possible (Norman et al., 2008). The advantage of the approach is that a relative location of UAV can be coupled with a specific role in the formation of UAVs or with a different setup of the field of view of on-board sensors. Also it can be used to reconFig. geometric relationships among UAVs in the formation. On the other hand, the approach of virtual structure requires precise position tracking and it synchronisation and this makes it susceptible to external disturbances. This is also related with high requirements of communication quality, which 
is crucial in computing desired positions. The precision of position tracking should be limited by placing dead zones around desired relative positions of UAVs in the structure, to avoid excitations of vibrations of control signals, when UAV is nearby its desired relative position. Then even small displacement of UAV position can generate high errors in angles of heading or pitch. The last approach uses only a peer-to-peer relation between a leader and a follower, or between two consecutive followers (Ambroziak and Gosiewski, 2014). In such case, this is relatively the simplest approach because interactions between UAV and information sharing are limited only to a pair of vehicles. The control algorithm in the leader-follower method is similar, but also simpler than this one used in the approach of the virtual structure. It comes from the fact that it is easier to control a distance and a heading angle than a relative position in the structure.

Other approaches in the field of multi-UAV systems are usually combinations of elements of these three basic approaches (Cai et al., 2012; Kownacki and Ołdziej, 2015). Each time, the main aim is to create an algorithm which will eliminate weaknesses of each of mentioned approaches. Therefore, also in this paper, we propose a method combining all three approaches: flocking behaviours, leader - follower and virtual structure. The main result of such combination is the increment of the formation stiffness, in respect to our previous work (Kownacki and Ołdziej, 2015). In the method, the leader of a UAVs formation still will be the one and only UAV that is controllable by the ground station. Hence, management of the formation should be easier and more convenient for the operator. Position and attitude of the leader determine also the origin and the orientation of a reference frame of the formation required to determine current positions of virtual migration points (nodes in the structure), which are assigned to each UAV. To make a UAV tracking its individual migration point as a relative position in the virtual structure, individual cohesion behaviours based on birds' flocks (Reynolds, 1987) can be applied. The second flocking behaviours, which are used in the method, are repulsion behaviours which help to prevent collisions with a vehicle located in the preceding node of the structure. Such combination of decentralised behaviours with the virtual structure makes possible to create coordinated, collective and coherent flights based on a simple information flow inside the formation. Scheme for information flow can be based on peer-to-peer transmission between pairs of radio modems. It is a simpler approach to information sharing in contrast to the position errors synchronisation, where UAVs must know positions errors of other UAVs (Norman et al., 2008). The case of two vehicle does not show this issue, which probably could occur during algorithm implementation. Moreover, as it was proved in (Ambroziak and Gosiewski, 2014), tracking of reference positions based on the minimization of positions errors will fail when those and headings errors are relatively large. Then UAVs will be unable to track their desired positions, which move in accordance with the leader or a virtual leader. The usage of flocking behaviours eliminates this problem and simultaneously it does not require applying two-stage switching control as it was in Ambroziak and Gosiewski, (2014). In the proposed method, UAV uses set-point values of pitch and heading, which arise from the orientation of the UAV position relative to the assigned position in the structure, and position error as displacement is minimised by the control of airspeed. An idea of combining virtual structure with behaviours is also presented in Cai et al., (2012), but in contrast to our conception, it applies only single behaviour of obstacle avoidance.

The remainder part of this paper is split into three sections. In the section titled "model of UAVs formation", we define the virtual structure of the formation and next describe relations between UAVs. We formulate also rules of local behaviours and present the way of information sharing which is essential for tracking of relative positions. In the next section, we present results of numerical simulations and a discussion on it. The last section offers conclusions and summarises the paper.

\section{STRUCTURE OF UAVS FORMATION}

Similarly to other research on the methods of virtual structure, the formation is treated as a rigid body. The position of each UAV inside the virtual structure is typically defined in a local frame, with respect to a reference point in the structure, which could be also the origin of the frame. In our case, the relative positions of the vehicles inside the virtual structure are defined in a reference frame $L$ whose the origin is located at the leader's position. Hence, the leader becomes a reference point for each UAV in the formation. In accordance to the trajectory of the leader, the virtual structure evolves in time and the desired relative positions for each UAV are determined by pre-set offsets from the current position of the leader. The diagram of the virtual structure and relations between desired positions $P_{i R}^{L}$ and $P_{i L}^{L}$ in the reference frame $L$ is presented in Fig. 1.

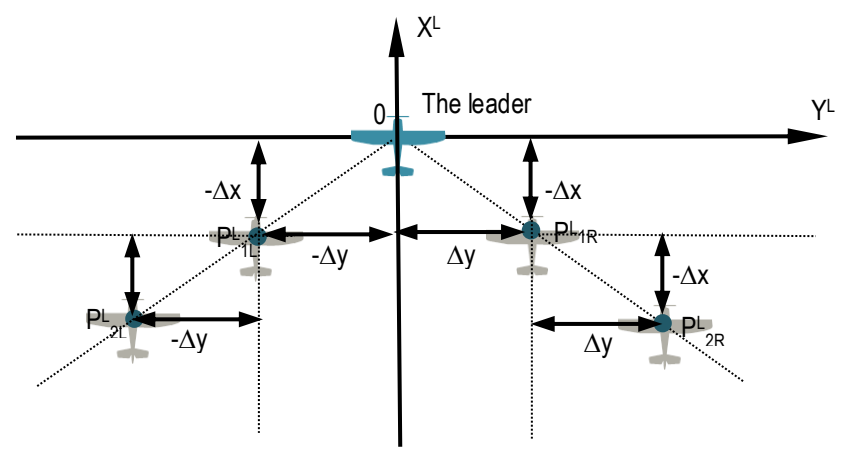

Fig. 1. A diagram of virtual structure in the reference frame $L$. $\Delta \mathrm{x}, \Delta \mathrm{y}-$ spacings between UAVs inside the virtual structure, $\mathrm{P}_{\mathrm{iR}(\mathrm{L})}^{\mathrm{L}}$ - the position of the $\mathrm{i}$-the UAV on the right or on left side of the leader

The proposed structure of the formation is identical as in a herd of ducks. We have two chains of UAVs as branches on both sides of the leader. In such structure, the position of each $\mathrm{UAV}$ in the formation frame $L$ (superscript) is defined by equations:

$P_{i R}^{L}=\left[\begin{array}{l}x_{i R}^{L} \\ y_{i R}^{L} \\ z_{i R}^{L}\end{array}\right]=\left[\begin{array}{c}-i \cdot \Delta x \\ i \cdot \Delta \mathrm{y} \\ 0\end{array}\right] \quad P_{i L}^{L}=\left[\begin{array}{l}x_{i L}^{L} \\ y_{i L}^{L} \\ z_{i L}^{L}\end{array}\right]=\left[\begin{array}{c}-i \cdot \Delta x \\ -i \cdot \Delta \mathrm{y} \\ 0\end{array}\right]$

where: $\mathrm{i}$ - the order of UAVs in chain $\mathrm{L}$ or $\mathrm{R}$ with respect to the leader, $\Delta \mathrm{x}, \Delta \mathrm{y}-$ spacings between UAVs, respectively in $\mathrm{x}$ and $\mathrm{y}$ axes.

Because the structure of the formation is treated as a rigid body and the origin of the formation frame $L$ is located at the leader's position, any rotation of the leader should be reflected in rotations of the virtual structure in respect to the inertial frame. Therefore, the equations defining the relative positions of UAVs 
inside the virtual structure, given in inertial frame $G$, are:

$P_{i R}^{G}=P_{L D}^{G}+D_{G}^{L} \cdot P_{i R}^{L} \quad P_{i L}^{G}=P_{L D}^{G}+D_{G}^{L} \cdot P_{i L}^{L}$

where: $\mathrm{D}_{\mathrm{g}}^{\mathrm{L}}$ - rotation matrix defining rotations between the formation frame $\mathrm{L}$ and the inertial frame $\mathrm{G}, \mathrm{P}_{\mathrm{LD}}^{\mathrm{G}}$ - the position of the leader in the inertial frame $\mathrm{G}, \mathrm{P}_{\mathrm{iR}}^{\mathrm{G}}$ - the desired position of the $\mathrm{i}$-th UAV on the right side of the leader (R) in the inertial frame G, $\mathrm{P}_{\mathrm{iL}}^{\mathrm{G}}$ - the desired position of the i-th UAV on the left side of the leader $(\mathrm{L})$ in the inertial frame $\mathrm{G}$.

According to equation (2), to determine in time its own desired relative position, each UAV must collect information about the leader's position and its orientation.

If each UAV knows its current position from the GPS system and its own desired relative position in the inertial frame $G$, the UAV will able to minimize its position error defined as the distance between the desired and the current position. The simplest way is to use PID or PI regulators (Norman et al., 2008). But, as it was found in (Ambroziak and Gosiewski, 2014), position tracking, which is based only on position errors is ineffective, especially when position errors are high and the reference trajectory has a lot of turns. In that case, due to limited turn radius and the misalignment between the heading of UAV and the reference heading of the leader, position error will grow again even if it was zero. Therefore, we propose an algorithm which combines the position error control with the heading angle control, to direct UAV towards its desired relative position in the structure.

\section{CONTROL ALGORITHM}

One of the methods, which can be applied to achieve an effective position tracking, is the cohesion behavior in birds flocks (Reynolds, 1987). This behavior, named differently as the attraction rule, is frequently used in robotics to concentrate agents around the centroid of a swarm. But instead of the centroid, we are able to apply this rule to any virtual point. Moreover, the point of aggregation can be different for each UAV in the formation. Hence, the nodes in the virtual structure defining desired relative positions of UAVs can be treated as virtual points which attract only their assigned agent. This also means that every agent implements its individual cohesion behavior. The idea of adapting cohesion behaviors of birds' flocks, as separate cohesion behaviors for every UAV in the formation, in the proposed approach is explained in Fig. 2.

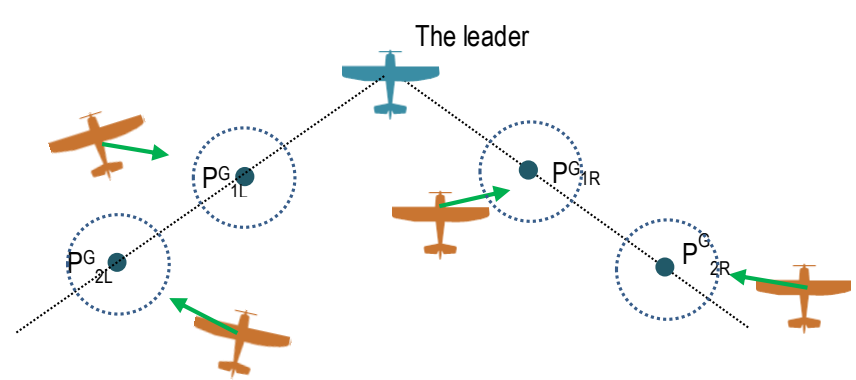

Fig. 2. An idea of position tracking in the virtual structure using individual cohesion behaviors, inspired by the theory of birds flocking. Green arrows define directions of cohesion. Each UAV has its assigned virtual point, which should attract it. Circles around virtual points in the structure define dead zones where cohesion behavior and speed control are not active
The principle of cohesion behavior is identical for every UAV (Fig. 3). To calculate the direction of cohesion that is defined jointly by desired heading and desired pitch angles, the knowledge about both the current position of the node and the current position of a UAV is required. The current position of every UAV is measured by GPS, and the current position of the node is the result of equation (2). Hence, the leader's position and his orientation in the inertial frame $G$ should be broadcasted to other UAVs through wireless communication network inside the formation. This issue will be discussed later. But back to the definition of the cohesion direction, it can be simply defined for the $i$-th UAV as a normalized vector $\overrightarrow{{ }^{C}{ }_{L L(R)}}$, which is given by equation (3). The vector can be named as a cohesion vector (Kownacki and Ołdziej, 2015).

$\overrightarrow{K^{C}{ }_{l L(R)}}=\frac{1}{\left|\overrightarrow{A P_{l L(R)}^{G}}\right|} \cdot \overrightarrow{A P_{l L(R)}^{G}} \quad \overrightarrow{A P_{L(R)}^{G}}=\overrightarrow{P_{l L(R)}^{G}}-\overrightarrow{P_{U A V_{l L(R)}}^{G}}$

where: $\overrightarrow{A P_{I L(R)}^{G}}$ - the cohesion vector is defined as a difference between the coordinates of the $i$-th $\mathrm{UAV}\left(\overrightarrow{\mathrm{P}_{\mathrm{UAV}}^{\mathrm{G}}(\mathrm{R})}\right)$ in the inertial frame $\mathrm{G}$ and the coordinates of assigned node in the virtual structure $\overrightarrow{P_{L(R)}^{G}},\left|\overrightarrow{A P_{I L(R)}^{G}}\right|-$ is a distance between the $i$-th UAV and the assigned node in the structure. This is also the displacement error.

The idea of cohesion behavior is illustrated in Fig. 3 .

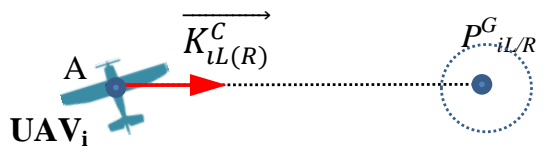

Fig. 3. An illustration of cohesion behavior. $P_{i L(R)}^{G}$ - the desired position in the structure for the $i$-th vehicle, $\overrightarrow{K_{l}}-$ the direction of cohesion

However, determining the direction of cohesion is not enough to achieve the effective position tracking, because it does not take into account one important parameter i.e. the displacement error. A separate control loop of airspeed is required to minimize this error. The easiest way to implement this is the usage of standard PID regulator, which will determine a desired airspeed to minimize the displacement error. The displacement error is defined as distance $\left|\overrightarrow{A P_{L(R)}^{G}}\right|$. Therefore, the desired airspeed for the i-th $U A V$ is defined by equation:

$S_{i L(R)}^{d}=K_{p} \cdot\left[\left|\overrightarrow{A P_{l L(R)}^{G}}\right|+K_{I} \cdot \int_{0}^{t}\left|\overrightarrow{A P_{l L(R)}^{G}}\right| d \tau+K_{D} \cdot \frac{d\left|\overrightarrow{A P_{L L(R)}^{G}}\right|}{d t}\right]$

where: $K_{p}, K_{D}, K_{I}$ - gains of PID terms, $\left|\overrightarrow{A P_{I L(R)}^{G}}\right|-$ the distance between the i-th UAV and the assigned node in the structure (the displacement error), $S_{i L(R)}^{d}$ - the desired airspeed of the i-th UAV.

To avoid instability, i.e. fluctuations of values of desired heading and desired pitch, which can be generated by rapid changes in the relative orientation between the current position of a UAV and the current position of the assigned node. This could happen while the displacement error is nearly zero. Therefore, dead zones should be defined as spheres around each node in the structure of the formation (Fig. 2). Inside dead zones, each UAV aligns its airspeed to the airspeed of the formation, i.e. to the airspeed of leader. Thus, a modified definition of the desired airspeed can be given by: 
$S_{i L(R)}^{d}= \begin{cases}P I D\left(\left|\overrightarrow{A P_{L L(R)}^{G}}\right|\right) & \left|\overrightarrow{A P_{L L(R)}^{G}}\right| \geq R \\ S_{i} & \left|\overrightarrow{A P_{L L(R)}^{G}}\right|<R\end{cases}$

where: $S_{i}$ - the airspeed of the entire formation (the leader's commanded airspeed), $R$ - a radius of dead zones around the nodes in the virtual structure, $S_{i L(R)}^{d}$ - the desired airspeed of the i-th vehicle, $\left|\overrightarrow{A P_{l L(R)}^{G}}\right|$ - the distance between the i-th vehicle and its assigned node.

Gains of PID terms should be adjusted in the way ensuring that the desired speed will be greater than the speed $S_{i}$ if UAV is outside the area of related dead zone. In that way, a UAV will be able to catch its assigned node in the structure with tolerance about $R$.

Collision avoidance between different UAVs in the formation should be also considered in the algorithm. It is necessary because of the fact that even wider spacing between the nodes in the virtual structure is not able to exclude a risk of collisions. Making maneuvers by the entire formation is related with a problem of intersections of trajectories that could be caused by inertia in dynamics of the position tracking. To prevent this, we propose a simple mechanism that is based on another flocking behavior, i.e. repulsion. This behavior is also implemented by the local control of a UAV, and its task is to repulse a UAV only from the preceding UAV in the structure. This way, the amount of information, which should be shared in the formation, is limited, and repulsion behaviors related with each pair of closest UAVs create a sequence of interactions, which will increase spacing between UAVs sequentially. The implementation of repulsion behaviors in the proposed algorithm is presented in Fig. 4.

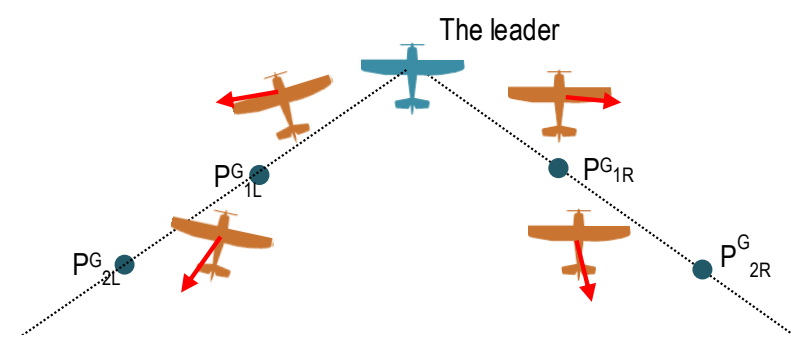

Fig. 4. Individual repulsion behaviors in the formation. Each vehicle is repulsed from the vehicle, which is preceding in the structure. It results in a chain of reactions. Red arrows represent directions of repulsion

The direction of the repulsion behavior is defined as it is shown in Fig. 5.

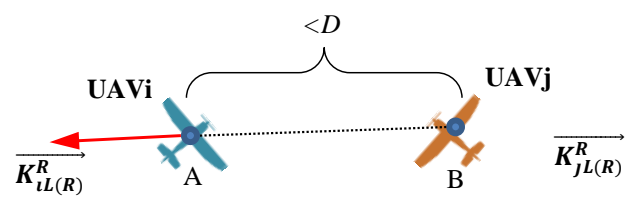

Fig. 5. An illustration of repulsion behavior. $\overrightarrow{\mathrm{K}_{\mathrm{L}(\mathrm{R})}^{\mathrm{R}}}$ - the direction of repulsion

In Fig. 5, a normalized vector $\overrightarrow{K_{l L(R)}^{R}}$ defines the direction of repulsion which is indispensable to keep a distance between two UAVs above the value of $D$. The repulsion vector is defined together by equations 6 and 7 .
$\overrightarrow{K_{L L(R)}^{R}}=\left\{\begin{array}{cl}\frac{1}{\left|\overrightarrow{P_{i L(R)}^{G} P_{j L(R)}^{G}}\right|} \cdot \overrightarrow{P_{i L(R)}^{G} P_{j L(R)}^{G}} & \left|\overrightarrow{P_{i L(R)}^{G} P_{j L(R)}^{G}}\right| \leq D \\ 0 & \left|\overrightarrow{P_{i L(R)}^{G} P_{j L(R)}^{G}}\right|>D\end{array}\right.$

$\overrightarrow{P_{l L(R)}^{G} P_{J L(R)}^{G}}=\overrightarrow{P_{l L(R)}^{G}}-\overrightarrow{P_{J L(R)}^{G}}$

where: $\overrightarrow{P_{L L(R)}^{G}}, \overrightarrow{P_{J L(R)}^{G}}-$ positions respectively of the $i$-th and the $j$-th vehicle, where $j<i,\left|\overrightarrow{P_{l L(R)}^{G} P_{J L(R)}^{G}}\right|$ - a distance between UAVs, $D$ - the minimum permissible distance between two UAVs.

A combination of the repulsion vector and the cohesion vector gives a resultant direction satisfying goals of both tasks of control, i.e. the position tracking and the collision avoidance. This resultant direction is given by vector $\vec{K}_{l}$.

$\overrightarrow{K_{l L(R)}}=\overrightarrow{K_{l L(R)}^{R}}+\overrightarrow{K^{C}{ }_{l L(R)}}$

When the resultant vector $\overrightarrow{K_{l L(R)}}$ would be already calculated, the set-points for low-level control loops, i.e. the desired heading angle and the desired pitch angle, can be easily determined from following equations:

$\overrightarrow{K_{L L(R)}}=\left[\begin{array}{l}x_{i L(R)} \\ y_{i L(R)} \\ z_{i L(R)}\end{array}\right]$

$\Psi_{i L(R)}^{d}=\arctan \left(\frac{x_{i L(R)}}{y_{i L(R)}}\right)$,

$\Theta_{i L(R)}^{d}=\arctan \left(\frac{z_{i L(R)}}{\sqrt{x_{i L(R)^{2}}+y_{i L(R)}^{2}}}\right)$

where: $\overrightarrow{K_{L L(R)}}-$ a vector which defines the resultant direction of flight, $\Psi_{i L(R)}^{d}$ - the desired heading angle, $\Theta_{i L(R)}^{d}$ - the desired pitch angle.

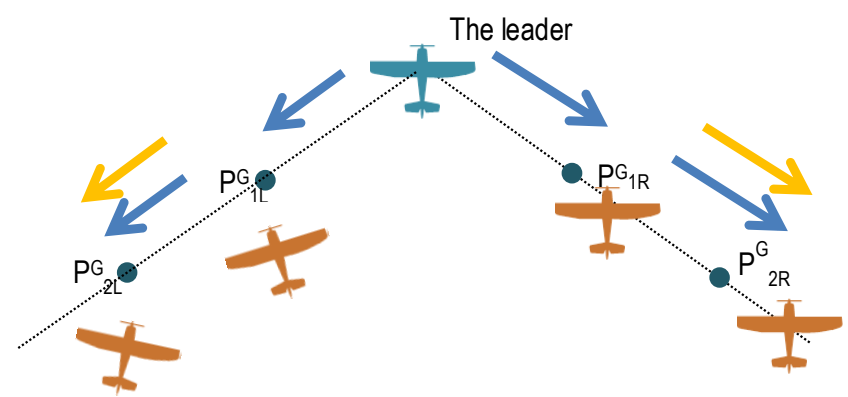

Fig. 6. Information flow inside the structure of the formation of UAVs, which is essential for the control. Blue arrows represent information about the leader; orange arrows represent information about the preceding UAV in the structure

Finally, we can formulate a control vector $\overrightarrow{u_{l}}$, which is composed with three parameters that are applied as set-points to the low-level control loops.

$\overrightarrow{u_{l}}=\left[\begin{array}{l}\Psi_{i L(R)}^{d} \\ \Theta_{i L(R)}^{d} \\ S_{i L(R)}^{d}\end{array}\right]$

As it was mentioned earlier, the algorithm requires information sharing between UAVs in the structure. Every UAV must collect 
Fig. 7 presents parallel trajectories of five UAVs creating the formation in "V" shape. While Fig. 8 shows horizontal projections of these trajectories. Positions of the UAVs at the end of the trajectories indicate clearly the shape of the formation.

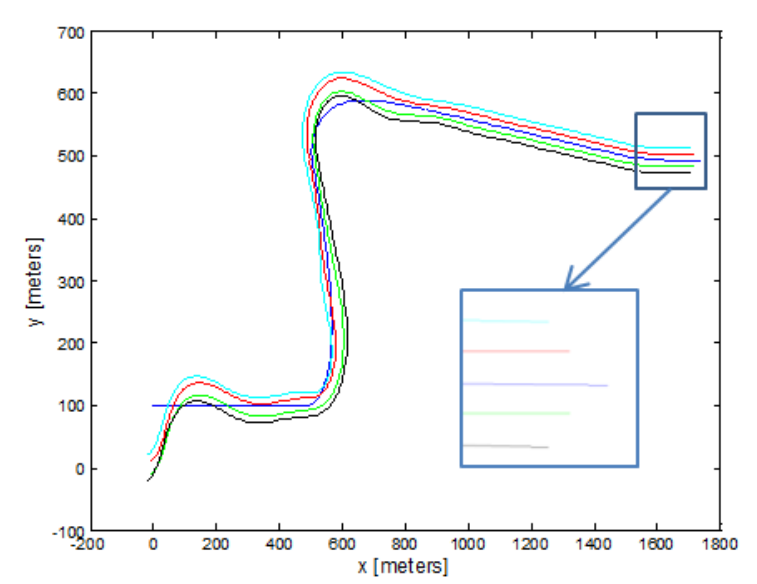

Fig. 8. Horizontal projections of the trajectories from Fig. 7

In next Figs. 9-12, there are respectively graphs of airspeeds, heading angles and pitch angles of all UAV, and finally distances between UAVs and their assigned nodes in the structure. The airspeed, the pitch and the heading of the leader are given in blue color.

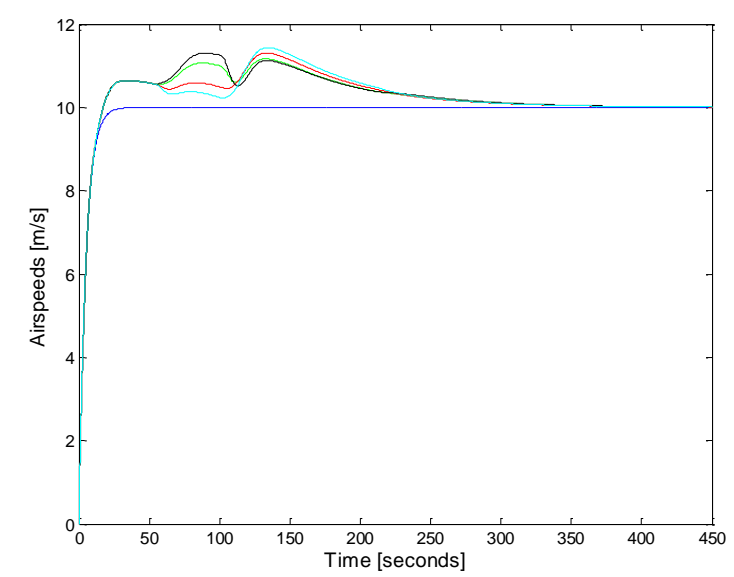

Fig. 9. Airspeeds of five UAVs in the formation. The airspeed of the leader is in blue

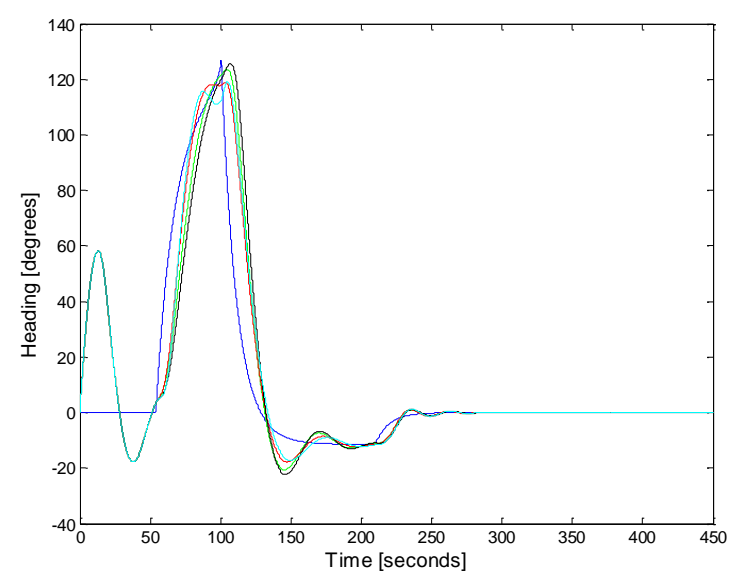

Fig. 10. Headings of five UAVs in the formation. The heading angle of the leader is in blue 


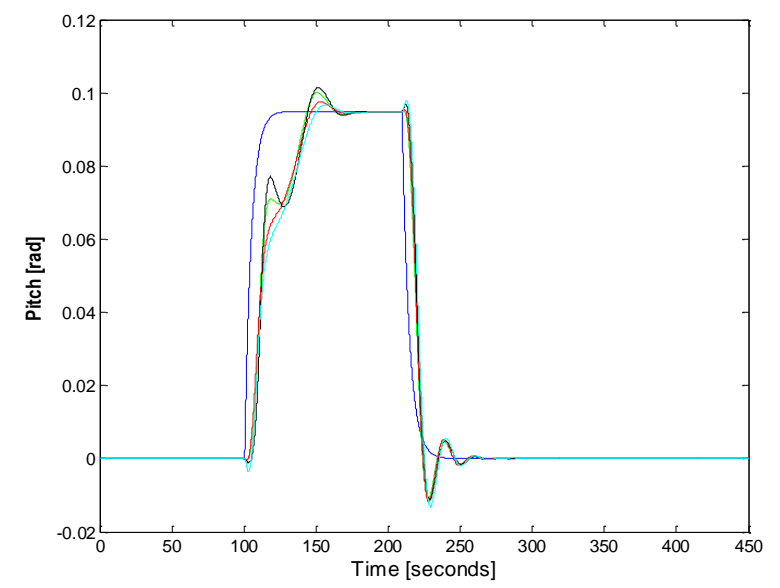

Fig. 11. Pitch angles of five UAVs in the formation. The pitch angle of the leader is in blue

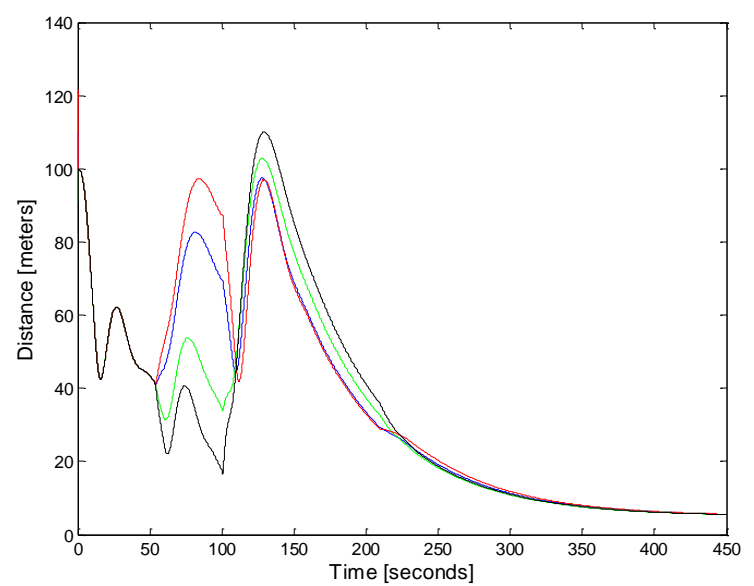

Fig. 12. Distances between UAVs and their assigned positions in the structure of the formation (Fig. 7 and 8)

Basing on Figs. 9 to 11 , it can be found that all four UAVs align their trajectories to the leader's trajectory pretty well. Of course, inertia in responses of followers is noticeable, especially in situations when the leader changes its flight direction (Fig. 10 and 11). Moreover, it can be noticed that the trajectory of the leader and the trajectories of the rest of formation are not parallel during manoeuvres. This is a synergistic result of the limited turning radius of fixed-wing UAVs, which is a square function of airspeed (Eq. 13), and depends on delays of information sharing and rotations of the local frame $\mathrm{L}$ around the leader's position in reference to the inertial frame $\mathrm{G}$.

$R=\frac{V^{2}}{g \cdot \tan (\phi)}$

where: $V$ - airspeed, $g$ - gravity acceleration, $\phi$ - limited roll angle.

Thus, if the leader turns to the right, the desired positions of the followers are shifted to the left in reference to their current positions and therefore, those UAVs turn to the left, oppositely to the leader. To minimize increased displacements in the positions (Fig. 12), the airspeeds of the followers should grow in reference to the leader (Fig. 9). Increased airspeeds result in longer turning radiuses, what can be visible as wider arcs of trajectories in comparison to the leader. At the ends of the sections of the trajectories, which are straight lines, UAVs manage to achieve the minimum displacement error that equals the radius of the dead zones. Not parallel trajectories can be observed regardless to the air- speed of UAVs and to the radius of the dead zones, what is shown in Fig. 13 and 14. This time, the airspeed is only $5 \mathrm{~m} / \mathrm{s}$ and the radius of dead zones is 2 meters.

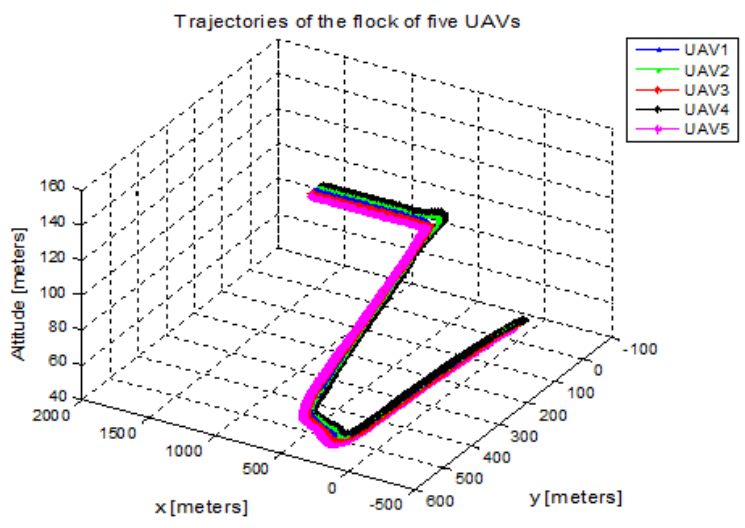

Fig. 13. Trajectories of simulated flight of the formation of five UAVs

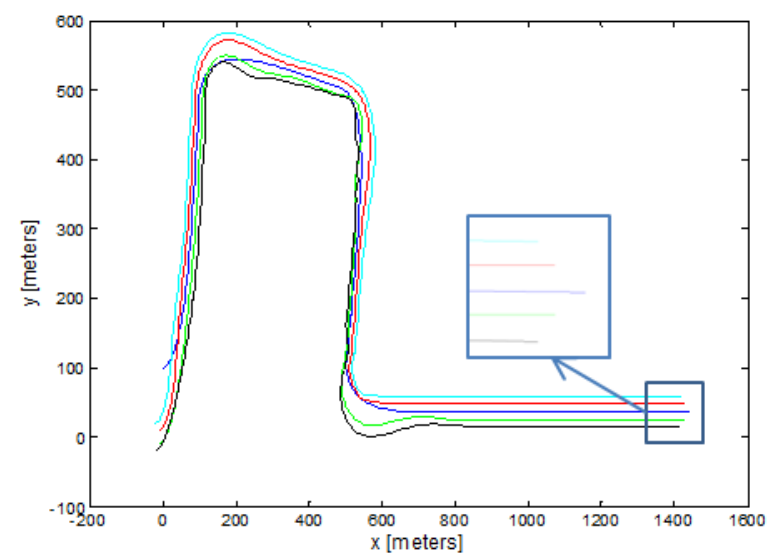

Fig. 14. Horizontal projections of the trajectories from Fig. 13

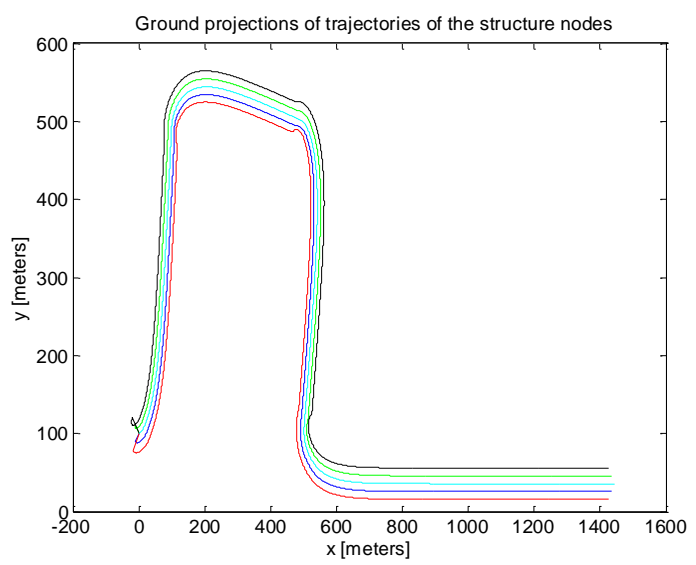

Fig. 15. Horizontal projections of the trajectories of the structure nodes which are related to the trajectories from Fig. 14

In Fig. 15, the trajectories of the nodes, i.e. desired positions, in the structure present an evidence for the effect of rotations of the virtual structure in reference to the inertial frame $G$, which are related with the leader's manoeuvres. Trajectories of the followers are bent in the reverse direction in respect to the trajectory of the leader, only later they go in the same direction. The effect is more visible for UAVs whose desired positions are located at longer distances from the leader. Spacing between vertical 
sections of trajectories of the nodes look different than those between horizontal sections due to different scales of $x$-axis and for $y$-axis.

In Fig. 14, we can observe how trajectories intersect and overlap, what could mean that there are collisions. To dispel concerns associated with this issue, Fig. 16 presents graphs of distances between all UAVs in the formation. All distances are always greater than zero and this excludes definitely the possibility of collisions. The closeness of the trajectories lines is only an illusion also caused by different scales for $x$-axis and for $y$-axis.

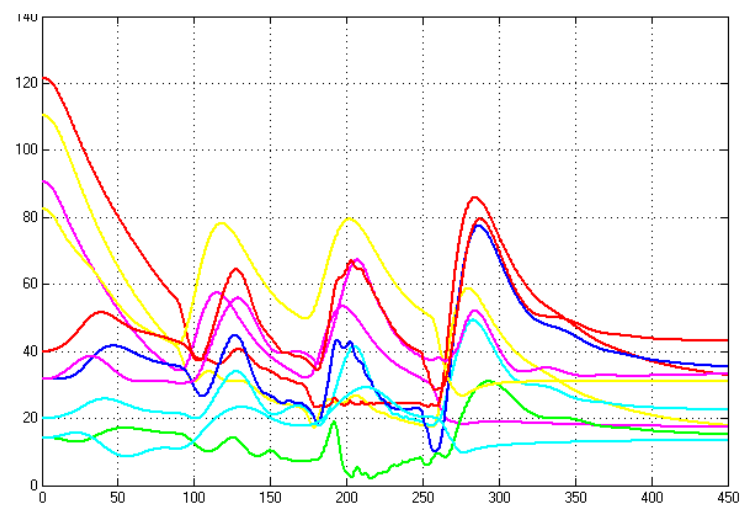

Fig. 16. Distances between all UAVs in the formation related with the trajectories from Fig. 13

Thus, the conclusion is that a simple collision avoidance rule, based on the repulsion behaviour is sufficient to provide safe flights of several UAVs in the proposed approach. The combination of behaviours of cohesion and repulsion offers a coherent and safe flight of the formation of UAVs.

The last issue, which should be discussed here, are delays in information flow between UAVs. If we take into account the fact that a UAV requires information from the leader to determine a current location of the node in the structure assigned to it, we will find that any delay in delivery of this information should impact only on relations between nodes. In other words, delays will increase real distances between nodes, but does not make local control unstable. To prove this argument, we prepared two simulations with different delays in the communication between two neighbours in the formation. In the second simulation, a value of delay is about 5 seconds, and it is greater hundred times than in the first case. Distances between UAVs and distances between UAVs and assigned nodes for each case are compared in Fig. 17 and Fig. 18.

In both Figs., UAV1 is the leader, UAV2 and UAV3 are just behind the leader respectively on its left and right side, UAV4 and UAV5 are behind UAV2 and UAV3, also respectively on the left and the right side of the leader. Basing on these results, it can be noticed that distances between pairs of UAVs in Fig. 17(2) are relatively greater than distances in Fig. 17(1), and this is the effect of increased delay. Simultaneously, distances between UAVs and their assigned positions in the formation look similarly on both sub Figs. of Fig. 18. It means that position errors used in the local control of UAV are insensitive to delays in communication. The problem is typical and common for all methods of formation flight of UAV, where positions of UAVs are synchronized in respect to a real or virtual reference point.
1)

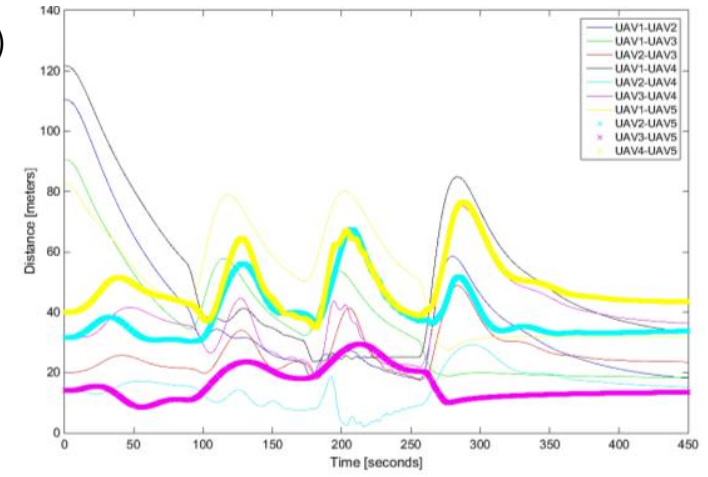

2)

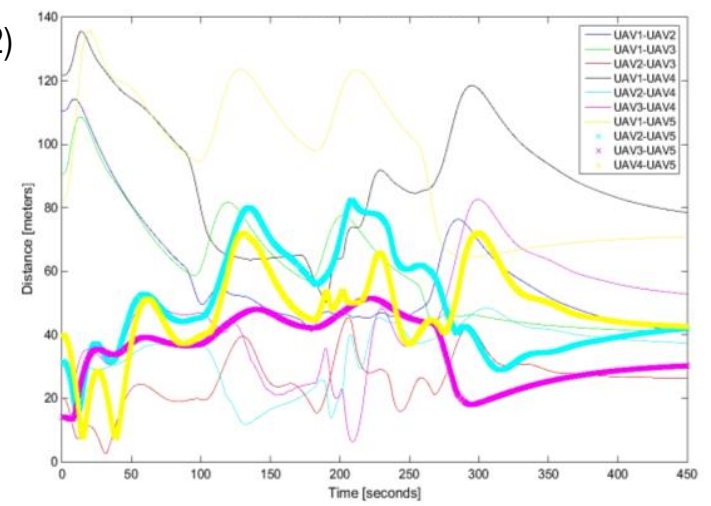

Fig. 17. Distances between all UAVs in the formation. 1) delay is about $0.05 \mathrm{~s}, 2$ ) delay is about $5 \mathrm{~s}$.

1)

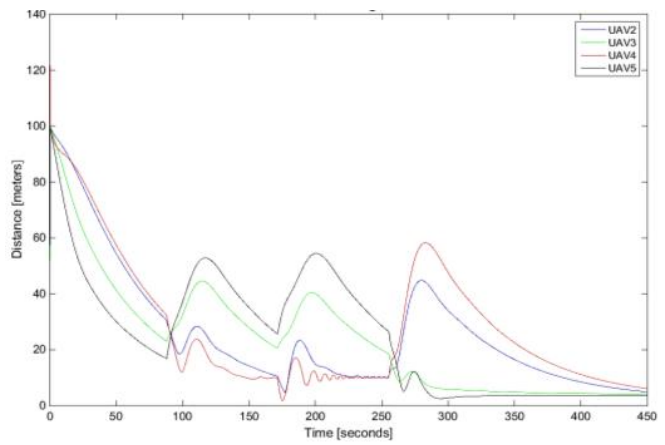

2)

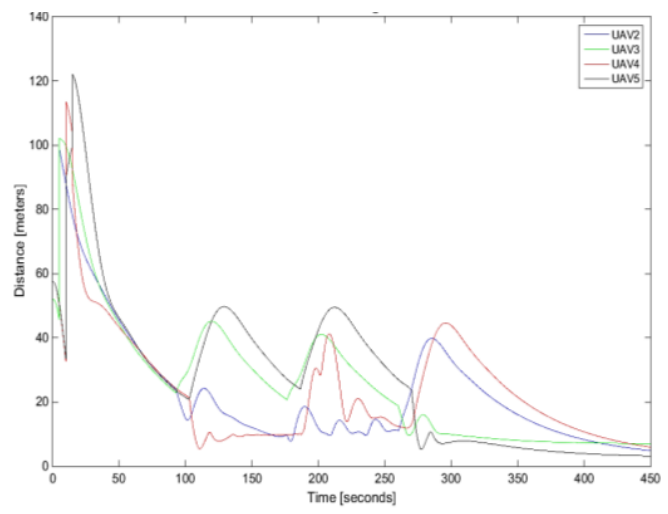

Fig. 18. Distances between UAVs and their assigned positions in the formation. 1) delay is about $0.05 \mathrm{~s}, 2$ ) delay is about $5 \mathrm{~s}$

\section{CONCLUSIONS}

In conclusion, the results of the simulations demonstrate the possibility of achieving a coherent formation flight by the combina- 
tion of following approaches: virtual structure, leader-follower and flocking behaviours. Presented approach can be an attractive way to implement formations of the UAVs in the real applications. Applying the real leader offers a convenient way to control formation flight missions by an operator. The behaviour of cohesion provides effective tracking of UAVs positions in the formation with the acceptable level of positions errors. Simultaneously, the simple implementation of the repulsion behaviour protects the structure of the formation against collisions between UAVs inside it, even if a UAV is repulsed only from the UAV which is preceding in the structure. Such implementation of the repulsion behavior reduces also the amount of information which should be shared between UAVs in the formation. Also in contrast to other approaches, information sharing in the proposed conception of formation control uses a simple communication method which could be based on a chain of peer-to-peer connections composed with standard radio modems. This approach to information sharing inside the formation increases the span of the formation because a spacing between two UAVs is limited only by the range of a single radio modem.

The found problem of trajectories, which are not parallel, is related with the inertia of dynamics of UAV, delays in the control and the communication, and also with rotations of the virtual structure around the reference point i.e. the leader. The problem can be solved in two ways, by resizing the structure during manoeuvres or alternatively by adjusting airspeeds of UAVs flying at the inner and outer side of a turn. This will be a subject of future research.

\section{REFERENCES}

1. Ambroziak L., Gosiewski Z. (2014), Two stage switching control for autonomous formation flight of Unmanned Aerial Vehicles, Aerospace Science and Technology, 46, 221-226.

2. Askari A., Mortazavi M., Talebi H.A. (2015), UAV Formation Control via Virtual Structure Approach, Journal of Aerospace Engineering, 28(1).

3. Cai D., Sun J., Wu S. (2012), UAVs Formation Flight Control Based on Behavior and Virtual Structure, AsiaSim 2012, Communications in Computer and Information Science, 325, 429-438.

4. Kownacki C., Ołdziej D. (2015), Flocking Algorithm for Fixed-Wing Unmanned Aerial Vehicles, Third CEAS Specialist Conference on Guidance, Navigation and Control, Advances in Aerospace Guidance, Navigation and Control, 415-431.
5. Low Ch. B., Ng Q.S. (2011), A flexible virtual structure formation keeping control for fixed-wing UAVs, 9th IEEE International Conference on Control and Automation, 621-626.

6. Norman H. M. Li, Hugh H.T. Liu (2008), Formation UAV Flight Control using Virtual Structure and Motion Synchronization, American Control Conference, 1782-1787.

7. Quintero S.A.P., Collins G.E., Hespanha J.P. (2013), Flocking with Fixed-Wing UAVs for Distributed Sensing: A Stochastic Optimal Control Approach, American Control Conference, 2025-2031.

8. Ren W., Beard R. W. (2004), Decentralized scheme for spacecraft formation flying via the virtual structure approach, Journal of Guidance, Control and Dynamics, 27(1), 73-82.

9. Reynolds, C.W. (1987), Flocks, herds and schools: a distributed behavioral model, ACM SIGGRAPH Computer Graphics, Proceedings of ACM SIGGRAPH '87, 25-34.

10. Seo J., Ahn Ch., Kim Y. (2009), Controller Design for UAV Formation Flight Using Consensus based Decentralized Approach, AIAA Infotech@Aerospace Conference Unmanned Unlimited Conference, 248-259.

11. Shan J., Liu H.T. (2005), Close-formation flight control with motion synchronization, Journal of Guidance, Control and Dynamics, 28(6), 1316-1320.

12. Shao Z., Zhu X., Zhou Z., Wang Y. (2014), A Nonlinear Control of 2-D UAVs Formation Keeping via Virtual Structures, Intelligent Robotics and Applications, Lecture Notes in Computer Science, 8917, 420-431.

13. Virágh Cs., Vásárhelyi G., Tarcai N., Szörényi T., Somorjai G., Nepusz T., Vicsek T. (2014), Flocking algorithm for autonomous flying robots, Bioinspiration \& Biomimetics, 9(2).

14. Xingping Ch., Serrani A., Ozbay H. (2003), Control of leaderfollower formations of terrestrial UAVs, Proceedings of 42nd IEEE Conference on Decision and Control, 498-503,

15. Yun B., Chen B.M., Lum K.Y., Lee T.H. (2008), A leader-follower formation flight control scheme for UAV helicopters, IEEE International Conference on Automation and Logistics, 1-3, 39-44. 